\title{
Validation and evaluation of measuring methods for the 3D documentation of external injuries in the field of forensic medicine
}

\author{
Ursula Buck ${ }^{1,2}$ \& Kirsten Buße ${ }^{1}$ \& Lorenzo Campana ${ }^{1,3}$ \& Christian Schyma ${ }^{1}$ \\ ${ }^{1}$ Institute of Forensic Medicine, University of Bern, Buehlstrasse 20, 3012 Bern, CH, Switzerland \\ ${ }^{2}$ Canton Police Bern, Accident service, Schermenweg 9, 3001 Bern, Switzerland \\ ${ }^{3}$ University Center of Legal Medicine Lausanne-Geneva, Chemin de la Vulliette 4, 1000 Lausanne 25, \\ Switzerland
}

Contact: Ursula Buck ursula.buck@irm.unibe.ch

\begin{abstract}
Three-dimensional (3D) measurement techniques are gaining importance in many areas. The latest developments brought more cost-effective, user-friendly, and faster technologies onto the market. Which 3D techniques are suitable in the field of forensic medicine and what are their advantages and disadvantages? This wide-ranging study evaluated and validated various 3D measurement techniques for the forensic requirements. High-tech methods as well as low-budget systems have been tested and compared in terms of accuracy, ease of use, expenditure of time, mobility, cost, necessary knowhow, and their limitations. Within this study, various commercial measuring systems of the different techniques were tested. Based on the first results, one measuring system was selected for each technique, which appeared to be the most suitable for the forensic application or is already established in forensic medicine. A body of a deceased, a face and an injury of a living person, and a shoe sole were recorded by 11 people with different professions and previous knowledge using the selected systems. The results were assessed and the personal experiences were evaluated using a questionnaire. In addition, precision investigations were carried out using test objects. The study shows that the hand-held scanner and photogrammetry are very suitable for the 3D documentation of forensic medical findings. Their moderate acquisition costs and easy operation could lead to more frequent application in forensic medicine in the future. For special applications, the stripe-light scanner still has its justification due to its high precision, the flexible application area, and the high reliability. The results show that, thanks to the technological advances, the 3D measurement technology will have more and more impact on the routine of the forensic medical examination.
\end{abstract}

\section{Keywords}

Forensic sciences, Patterned injuries, 3D documentation, Photogrammetry, Surface scanning 


\section{Introduction}

In forensic medicine, photogrammetry [1-8] or highly precise three-dimensional (3D) surface scanning $[7,9-11]$ is used for the true to scale and color documentation of external patterned injuries. These data of the body surface are fused with the 3D computed tomography and magnetic resonance imaging datasets by using specific radiographic markers $[10,12,13]$.

The generated 3D model of a body of a deceased can be virtually moved and matched with an injuryinflicting tool in order to reconstruct courses of crime and accident [14-20]. However, up to now, only a few institutes use 3D documentation methods such as photogrammetry or 3D scanning methods. External patterned injuries are often still documented in the conventional way (photograph with scale). The reason can be the high-cost equipment and the advanced knowledge required for the application of this technique.

In recent years, lots of new 3D documentation methods have been made available on the market. The new methods are easier to use and cheaper and the documentation is faster [21]. Individuals with less experience in 3D technology can easily apply the methods. This development simplifies the use of 3D documentation in many areas, including forensics. 3D scanning methods are becoming more important for the police. Photogrammetry and 3D laser scanning are used for the documentation of accidents and crime scenes. Which 3D measuring techniques are useful for forensic medicine and what are the advantages and disadvantages?

This study has the goal to validate and evaluate the usefulness of 3D measuring techniques for forensic medicine. The advantages and disadvantages of the different techniques, from high-tech methods to low-budget systems, are assessed, particularly with regard to accuracy, user-friendliness, expenditure of time, mobility, costs, necessary knowhow, and limitations.

\section{Material and methods}

In the following study, different objects were documented in 3D: a whole body of a deceased person (Fig. 1a), a face (Fig. 1b) and an injury (Fig. 1c) of a living person, and a sole of a shoe (Fig. 1d). Four different measuring techniques were used to document these 3D models true to scale and color. One commercial measuring system that seems best adapted for forensic application or is already established in forensic medicine was selected for each measuring technique. As a photogrammetrybased fringe-pattern projection system, the TRITOP/ATOS system (GOM, Braunschweig, Germany) [22], as hand-held scanner, the Go!Scan 50 (Creaform/ Ametek, Québec, Canada) [23], as photogrammetry system, the AGISOFT PhotoScan software (Agisoft, St. Petersburg, Russia) [24], and the low-budget scanner Kinect v2 (Microsoft, USA) were selected.

The four different objects were documented using these measuring systems by 11 persons with the following background and education: Three surveying engineers and two policemen with advanced experience in 3D documentation methods, a radiographer and an autopsy technician with basic experience, and two forensic pathologists and two policemen with no experience in using such methods.

Each person filled out a questionnaire about their individual experience with the different methods. Apart from the time needed for preparation, acquisition, and post-processing, they had to assess how satisfying the preparation was, how user-friendly the software and hardware were, how easy to learn, mobile, and prone to errors it was, and if there was a possibility to check the result on-site. 
These aspects were rated with a grade from one (highly satisfactory) to six (highly unsatisfactory). The systems which were difficult to learn in little time (max. $1 \mathrm{~h}$ ) were only used by the persons with basic or advanced experience. The forensic pathologists and policemen with no experience were assisted in using these systems.

Two test objects were created to check the accuracy. The first was a polystyrene head (Fig. 2a) measuring $20 \times 15 \times 24 \mathrm{~cm}$. This round object was used to compare the resolution and accuracy of the shape and to determine misclosures, which can occur in an all-around scan of an object. In order to test the accuracy of length, six polystyrene spheres with a diameter of about $4 \mathrm{~cm}$ were attached to a wall with an approximate distance of $1 \mathrm{~m}$ between them (Fig. $2 \mathrm{~b}$ ). This resulted in a measured distance of about $5 \mathrm{~m}$. Round black-and white points were attached to both test objects. These test objects were only scanned by the surveying engineers. The accuracy check was performed in the GOM ATOS Professional software.

Additionally, the hand-held scanner Space Spider (Artec 3D, Luxembourg) [25] was tested to document the sole of a shoe because of its higher resolution compared to the Go!Scan 50. It was not selected for the whole evaluation though because the measuring range is too small for whole body documentation and the light is too bright for living persons. To show the possibilities of a computed tomography (CT) scanner, it was used for the documentation of the sole of the shoe. Because of the missing color information, it was not useful for the other objects.

The used measuring systems are described below:

\section{Stripe-light scanner (GOM TRITOP/ATOS) (Fig. 3a)}

To create a high-resolution 3D surface model, the ATOS III scanning system was used. The structuredlight 3D scanner consists of a projector unit in the center and two digital cameras left and right, each of which was equipped with a 4-million pixel CCD sensor. The projector unit projects a striped light pattern on the surface while the two cameras record images. Since the distance between the two cameras and the angle between them are known, the software calculates the 3D coordinates of each sensor's pixel points using the triangulation approach. Up to 4 million 3D surface points are determined in each scan. Through the non-coded reference points, the individual scans are merged automatically into one dataset. The recently scanned model as well as the position of the scanner for the next scan is shown on the computer in real time.

Photogrammetry using the TRITOP system is necessary for the automatic texture mapping of the 3D surface scan model. It was only applied for the documentation of the whole body of a deceased. Hereby, the body was recorded with a DSLR camera (Nikon D2X, Nikon, Inc., Tokyo, Japan) from various different angles and elevations. Plastic crosses covered with coded reference points and two coded scale bars were placed near the area to be recorded. The software merged the photographs together using these coded reference points and calculated a bundle block adjustment internally.

Only the surface scanner was used for the documentation of a living person and the sole of a shoe. For texture mapping of the 3D model of a living person, a photograph was projected onto the surface model using reference markers.

\section{Hand-held scanner (CREAFORM Go!Scan 50) (Fig. 3b)}

This hand-held scanner Go!Scan 50 is also based on structured light. The scanner emits a white light pattern, similar to a QR Code. Two cameras observe the distortion of the pattern on the scanned 
object. A third camera records the color information of the calculated 3D points. The scanner works with a rate of 550,000 measurements per second and a scanning area of $380 \times 380 \mathrm{~mm}$ with a resolution of $0.5 \mathrm{~mm}$ and a point accuracy of up to $0.1 \mathrm{~mm}$, according to the manufacturer. The surface is captured while moving the hand-held scanner over the object. A lamp guidance system helps to find the correct scanning distance. The recommended size of objects to be scanned is 0.3 to $3 \mathrm{~m}[23]$.

During scanning, the Go!Scan 50 was connected to a laptop which was necessary for controlling the scanner, but it also showed the recently scanned models in real time. This provided the possibility to check the completeness of the 3D model while scanning and to fill lacking areas by continuing scanning.

\section{Hand-held scanner (Artec 3D Space Spider) (Fig, 4a)}

The recording method of the Space Spider is similar to the Go!Scan 50. The 3D point resolution of the result is up to $0.1 \mathrm{~mm}$ and the 3D point accuracy is up to $0.05 \mathrm{~mm}$, but the scanning volume is limited to $2000 \mathrm{~cm}^{3}$ according to the manufacturer [25]. That makes the Space Spider useful for small objects which should be digitized in high-resolution geometry and color.

\section{Photogrammetry (AGISOFT PhotoScan) (Fig. 3c)}

PhotoScan is a photogrammetry software for automatic bundle block adjustment and generation of 3D models from photos of calibrated and non-calibrated cameras. The photos for the photogrammetric models in this study were taken with an uncalibrated DSLR camera (Nikon D700, Nikon, Inc., Tokyo, Japan) with a zoom lens between 24 and $70 \mathrm{~mm}$. The area to be documented was photographed in overlapping rows with a constant focal length and distance to the object. The photos were taken in overlaps of at least $80 \%$ [26].

\section{Low-budget scanner (Microsoft Kinect v2 device)}

The Kinect device was developed by Microsoft in cooperation with the company PrimeSense (Israel) as hardware for controlling the video game console Xbox 360 and was one of the first low-cost 3D scanners on the market. The Kinect v2 device consists of an infrared sensor with $512 \times 424$ pixels and a HD wide-range camera with a resolution of $1920 \times 1080$ pixels and 30 frames per second. An infrared projector (IR) illuminates the scene while the IR camera receives the deepness and image information in real time [27].

\section{CT scanner (Siemens Medical Somatom Definition AS) (Fig. 4b)}

The examination of the shoes was performed with a sixty-four-detector row system (Somatom Definition AS, Siemens Medical, Erlangen, Germany) with the following raw data acquisition parameters: $140 \mathrm{kV}$, Caredose $\mathrm{mAS}$ and $0.4 \mathrm{~mm}$ collimation and the following image reconstruction parameters: thickness $0.4 \mathrm{~mm}$, increment $0.2 \mathrm{~mm}$, FoV $112 \mathrm{~mm}$, and reconstruction kernel B70s, extended CT-Scale. The surface rendering was performed in the OsiriX software.

\section{Results}

3D models of the body of the deceased and the injury and the face of a living person were generated based on the data from the stripe-light scanner, the hand-held scanner, and the photogrammetry and exported as .stl- (without color) and .obj- (with color) files. Figure 5 shows the 3D models 
without color of the body of the deceased, and Fig. 6 shows the 3Dmodels without and with color of the faces.

The shoes were additionally documented with the high resolution hand-held scanner Space Spider and a CT. The resulting 3D models of the shoe are shown in Figs. 7 and 8.

To verify the quality (resolution and accuracy) of the 3D models obtained from the different measuring techniques, two test objects were measured and the deviations were analyzed. The stripe-light scanner was used as reference because of its higher resolution and accuracy in comparison with the other evaluated techniques.

\section{Round test object}

Mesh deviations were performed between the different models of the round test object created using the same system as well as between the models obtained from using the stripe-light scanner and those of the hand-held scanner, photogrammetry, and Kinect device. The results are shown in Fig. 9 as false color image. It can be seen that the deviations between the results of the stripe-light scanner and the hand-held scanner (Fig. 9b) are minimal. The model of the difference between stripe-light scanner and photogrammetry result (Fig. 9c) shows deviations up to $1 \mathrm{~mm}$. The largest deviation was located under the chin; smaller deviations were located in the area of the lips, eyes, and on top of the head. The shape of the face in general matched very well and only small deviations under $0.1 \mathrm{~mm}$ could be seen. The result of the Kinect device (Fig. 9d) presented large deviations up to $10 \mathrm{~mm}$ with regard to the width and height of the head.

\section{Long test object}

The distances between the attached spheres and points were measured in the models generated by the different systems. The standard deviation of the long test object ( $5 \mathrm{~m}$ distance) was $\pm 0.3 \mathrm{~mm}$ for the stripe-light scanner, $\pm 0.43 \mathrm{~mm}$ for the hand-held scanner, and $\pm 2.05 \mathrm{~mm}$ for the photogrammetry. The standard deviation of the hand-held scanner in comparison to the stripe-light scanner was $\pm 1.33 \mathrm{~mm}$, and the standard deviation of the photogrammetry in comparison to the stripe-light scanner was $\pm 2.54 \mathrm{~mm}$.

\section{Questionnaire}

The questionnaire filled out by the 11 test persons was evaluated. The diagram in Fig. 10 shows the time expenditure for the different systems. In Fig. 11, the results regarding preparation, software, hardware, learnability, mobility, proneness to error, and controllability are presented.

\section{Discussion}

Measuring techniques which seemed to be useful in the forensic field were evaluated. For each measuring technique, one commercial system was selected. As stripe-light technique, the TRITOP/ATOS system from GOM was used. This scanning system has been applied in forensic sciences since 2003. As hand-held scanner, the Go!Scan 50 from Creaform was chosen because of its user-friendliness, reliability, accuracy, and flexibility. An important condition in the preselection of the hand-held scanner was that it was able to document an entire body true to color within a short period. Hand-held scanners with higher resolution were also evaluated, for example, Space Spider from Artec3D, but this scanner is only suitable for small objects and was therefore not considered in 
the whole comparison. The CT was also used just for the sole of the shoe to show the possibilities if it is available. For the other study objects, it is not useful because of the missing color information.

When selecting a photogrammetric evaluation software, AGISOFT PhotoScan was chosen because of its ease of use, providing good results and low purchase price.

Another possibility consists attaching radiological reference markers around the injury, performing a CT scan and a close-up photo for the texture mapping of the 3D model of the injury [13]. This method was not integrated in this study because it is not useful for the whole body. Measuring methods using laser beams, for example, ViuScan from Creaform, were not integrated in this study, because the first results showed that they are not as useful as the other methods for this forensic approach. However, for the documentation of incident scenes, terrestrial laser scanning is of course a very important method.

In the following part, the scan results from the different chosen measuring systems will be discussed.

\section{Body of a deceased}

Figure 5 shows the different scan results generated from the data of the stripe-light scanner (Fig. 5a), the hand-held scanner (Fig. 5b), and the photogrammetry (Fig. 5c) with a detailed view of the back of the body. Especially in the latter, the differences in resolution and level of detail are clearly visible. The skin lesion is clearly visible in the result from stripe-light scanner (Fig. 5a), while the skin lesion is blurred in the result from the hand-held scanner (Fig. 5b) and is not clearly visible in the photogrammetry result (Fig. 5c). A general problem can also be observed in this figure: hair is very difficult to capture (e.g., Fig. 5a, b and 6a, b). This problem occurs mostly with scalp hair, but, depending on its density and color, also with, e.g., beards, chest hair, and eyebrows.

\section{Face and injury of a living person}

The scan of living persons has to be quick, because otherwise the movement artefacts become too large. The results of different faces are shown in Fig. 6 . The geometry of the stripe-light scanner model (Fig. 6a) is highly detailed; for textured data, a photo has to be projected on the 3D model. The geometry of the hand-held scanner model (Fig. 6b) is less detailed than the model from the stripe-light scanner but still shows all relevant features. The texture resolution is not so good but can be improved by projecting a photograph on it. The geometry of the photogrammetry model (Fig. 6c) has a lower resolution but the photorealistic resolution of the texture is very good.

\section{Sole of a shoe}

On the sole of the shoe (EUR size 38), the differences in resulting geometry between the methods are clearly visible (Figs. 7 and 8). For a morphometrical comparison, detailed 3D models are necessary. The result from the stripe-light scanner shows a very detailed shoe profile (Fig. 7a). The result from the CT scanner is not much worse (Fig. 7d). The 3D models from the photogrammetry and Kinect scanner are not usable for any morphometrical purposes (Fig. 7b, e). In the detailed view of the shoe sole in Fig. 8, the main differences between the stripe-light scanner, the CT scanner, and two hand-held scanners are shown. The results with sharp edges and patterns on the block elements are from the stripe-light scanner and the high-resolution hand-held scanner for small objects (Space Spider) (Fig. 8a, d). For small objects, the comparable resolution, accuracy, and attention to detail make the Space Spider a cheaper alternative to the expensive ATOS scanner. The differences to the result from the Go!Scan 50 (Fig. 8c), also a hand-held scanner but for larger objects, are clearly 
visible. The result from the CT scanner shows that the single block elements of the sole are nicely documented but the edges of the blocks are smooth (Fig. 8b).

With the scan results in mind, the different systems will be evaluated with their advantages and disadvantages in the following part.

\section{Stripe-light scanner (GOM TRITOP/ATOS)}

The 3D models of all objects created by means of the stripe-light scanner have high detail accuracy and resolution. As shown in Fig. 5a, small details of injuries are visible in the surface model and not only in the textured data (e.g., Fig. 1c). For the documentation of living persons, it is important that no movements occur during the scan. Furthermore, it is recommended to darken the room for an ideal scanning quality. The GOM Software is powerful and has useful functions for handling, modifying, and transforming even large scan data and 3D models. The 3D models can be exported as .stl-file (without color) and as .ply-file (with color) as well as many other formats.

Advantages of the stripe-light scanning method are the flexible measuring volumes and the high quality data.

Disadvantages are high costs (more than 50.000 euros) for the equipment and the limited mobility of the system. Furthermore, the method should be used by trained specialists for good results.

\section{Hand-held scanner (CREAFORM Go!Scan 50)}

Right after finishing the scan, the 3D model can easily be edited with the software and/or directly exported in common formats. It is not possible to export the model directly as 3D-pdf- file.

The advantage of the hand-held scanner is that even minimally trained users are able to quickly scan an object and create a detailed and textured 3D model.

The geometrical resolution of this scanner is sufficient for many applications; however, the texture resolution is not as good as the texture of the 3D model generated by PhotoScan (Fig. 6b, c).

The hand-held scanner is cheaper than the stripe-light scanner, but still costs between 15.000 and 30.000 euros.

\section{Photogrammetry (AGISOFT PhotoScan)}

In PhotoScan, the generation of a true-to-color 3D model is very easy and can be done halfautomatically. The resulting model has high-resolution color information; however, the quality of the geometry depends on the configuration of the photos around the object and the quality of the photos themselves. Especially in the border areas of the 3D model, the measuring noise is very high. In the case of the body, injury, and face, the color information is more import than the resolution of the surface. However, the surface resolution of the shoe sole (Fig. $7 \mathrm{~b}$ ) is not detailed enough and consequently not useful for 3D match analysis.

The true-to-color and true-to-scale 3D models can be exported in common formats. Additionally, it can be directly exported as 3D-pdf-file and opened on each computer with a current version of the Adobe Acrobat Reader. This is really useful to visualize and share the 3D data with people without knowledge in 3D computer software, or if special software is not available. 
The advantage of the photogrammetry is the high resolution color information that no electricity has to be available and the system is very mobile: Only a camera and a scale are necessary for the documentation of objects of all dimensions. The costs of the software are moderate (less than 5.000 euros). With the exception of the reduced geometry quality especially in the border areas, the results show high-resolution color information (Fig. 6c) which is very important in the field of forensic medicine. Disadvantages are that enough ambient light is necessary and that the quality of the resulting 3D model is user-dependent.

\section{Low-budget scanner (Microsoft Kinect v2 device)}

The differences between model and reality were not only seen in the evaluation but were already apparent during the visual comparison of the results (Fig. 7e and 9d). The resolution of the 3D geometry and color in the models were not satisfactory. That was the (main) reason not to further consider the Kinect.

To validate the different systems, an accuracy check was performed.

\section{Accuracy check}

The results of the round test object showed that the stripe-light scanner delivers the best geometric data (Fig. 9). The hand-held scanner has a lower resolution in detail but no significant deviation. The photogrammetry model has deviations up to $1 \mathrm{~mm}$ and the Kinect model has very high deviations. Therefore, if high-resolution surface data are required, the stripe-light scanner is the best choice. The hand-held scanner delivers accurate but less detailed data. With photogrammetry, some small deviations in the geometry can occur, but in many cases, the result is still accurate enough. The Kinect device delivers data which are not accurate enough for 3D match analysis or 3D reconstructions. Better, but still not comparable, results could be obtained using a calibrated Kinect scanner [27].

The analysis of the long test object showed that the stripe-light and hand-held scanners produce small deviations on long distances. Because the correct scaling of the object is important for the photogrammetry, scale bars of sufficient length have to be placed next to or on the object.

\section{Questionnaire}

As shown in Fig. 10, the time for preparation and acquisition for using photogrammetry and the hand-held scanner is short. The time for post-processing using the hand-held scanner is also very short and the results are available on scene, whereas the post-processing is more time-consuming with photogrammetry. The time needed for preparation and scanning with the stripe-light scanner is significantly longer while the post-processing is done rather quickly.

In the questionnaire with a scale from 1 (very satisfying) to 6 (not satisfying at all), the hand-held scanner received the best ratings (Fig. 11), especially for the preparation (1.1), user-friendly software (1.0) and hardware (1.5), simplicity ("easy to learn") (1.3), and error-proneness ("prone to errors") (1.0). The quality check of the scan result of the stripe-light scanner (1.2) and the hand-held scanner (1.3) was ensured. The current 3D model is shown on the computer and can be completed with additional scans until the 3D model is adequate. Only in the case of living people, a new scan is recommended if the result is not satisfactory, because the subject likely moved while the scan result is checked. With photogrammetry, the ability to inspect the scan result on-site is strongly limited which is reflected in the poorer rating 4.1 (medium). It would be time-consuming to do the photogrammetric calculations on-site on a laptop. Furthermore, it would be necessary that the user 
masters the software and is capable of correctly assessing the results. A high-performance workstation is recommended for the analysis. The photogrammetry was rated best for mobility (1.0) because only a camera and scale are necessary for the 3D documentation. The hand-held scanner requires electricity and a connected laptop (2.3). The stripe-light scanner was rated poorly for its mobility (4.2, heavy equipment and time-consuming setup and calibration of the sensor), the simplicity of learning its use (3.1), and its proneness to errors (3.5). For this reason, it is recommended that only trained specialists perform the scans with the stripe-light scanner.

\section{Conclusion}

Stripe-light scanner, hand-held scanner, and photogrammetry are suitable documentation methods for forensic sciences. The methods differ in resolution, accuracy, required technical knowledge, costs, weight of the equipment, and the time needed for documentation and analysis.

For most applications, the combination of two methods leads to the best results. Utilizing the good surface model from the hand-held scanner or the stripe-light scanner and projecting the high quality texture from the photogrammetry on it result in a 3D model with the advantages of both methods.

In the case of a body of a deceased, we recommend the combination of the hand-held scanner for a good geometrical resolution and photogrammetry for a high texture resolution. Together, they deliver good results within an acceptable time.

Documenting a face or an injury of living persons with two methods takes too much time for most victims or suspects to keep still. In this case, it is more suitable to select one method. If the color information and mobility are important, e.g., for injuries, we recommend photogrammetry. If the geometry of a face is important, for example for geometric comparisons [28], we recommend a hand-held scanner instead.

In the case of injury-inflicting instruments, the geometry is mostly more important than the color information of the surface. Therefore stripe-light scanning or high-resolution handheld scanning gives the best result.

\section{Acknowledgements}

The authors would like to thank Nicole Schwendener, Julia Brünig, Jeremias Klaus, André Lüthi, Gerhard Reichen, Daniel Leuenberger, Thomas Stöckli, and Michal Neukamp for testing the different methods and filling out the evaluation form.

The people behind the faces in Figs. 1 and 6 explicitly declared their consent for showing their undisguised faces within this publication. The author would like to thank them too.

\section{References}

1. Brueschweiler W, Braun M, Fuchser H, Dirnhofer R (1997) Photogrammetrische Auswertung von Haut- und Weichteilwunden sowie Knochenverletzungen zur Bestimmung des Tatwerkzeuges grundlegende Aspekte. Rechtsmedizin 7(3): 76-83. https://doi.org/10.1007/BF03042360 
2. Thali MJ, Braun M, Kneubuehl BP, Brueschweiler W, Vock P, Dirnhofer R (2000) Improved vision in forensic documentation: forensic, 3-D/CAD-supported photogrammetry of bodily injury external surfaces, combined with volumetric radiologic scanning of bodily injury internal structures, provides more investigative leads and stronger forensic evidence. P Soc Photo-Opt Ins 3905: 213-21. https://doi.org/10.1117/12.384876

3. Thali MJ, Braun M, Markwalder TH, Brueschweiler W, Zollinger U, Malik NJ, Yen K, Dirnhofer R (2003) Bite mark documentation and analysis: the forensic 3D/CAD supported photogrammetry approach. Forensic Sci Int 135(2):115-121. https://doi.org/10.1016/s0379-0738(03)00205-6

4. Thali MJ, Braun M, Brueschweiler W, Dirnhofer R (2003) 'Morphological imprint': determination of the injury-causing weapon from the wound morphology using forensic 3D/CAD supported photogrammetry. Forensic Sci Int 132(3):177-181. https://doi.org/10.1016/s0379-0738(03)00021-5

5. Buck U (2001) Anwendung der Photogrammetrie bei der Polizei des Landes Baden-Württemberg. Deutscher Verein für Vermessungswesen Heft 2:120-131

6. Villa C (2016) Forensic 3D documentation of skin injuries. Int J Legal Med 131(3):751-759. https://doi.org/10.1007/s00414-016-1499-9

7. Urbanova P, Hejna P, Jurda M (2015) Testing photogrammetry based techniques for threedimensional surface documentation in forensic pathology. Forensic Sci Int 250:77-86. https://doi.org/10.1016/j.forsciint.2015.03.005

8. Luhmann T, Robson S, Kyle S, Boehm J (2014) Close-range photogrammetry and 3D imaging. Walter de Gruyter

9. Subke J, Wehner HD, Wehner F, Szczepaniak S (2000) Streifenlichttopometrie (SLT): a new method for the three-dimensional photorealistic forensic documentation in colour. Forensic Sci Int 113(13):289-295. https://doi.org/10.1016/S0379-0738(00)00236-X

10. Thali MJ, Braun M, Wirth J, Vock P, Dirnhofer R (2003) 3D surface and body documentation in forensic medicine: 3-D/CAD photogrammetry merged with 3D radiological scanning. J Forensic Sci

11. Thali MJ, Braun M, Dirnhofer R (2003) Optical 3D surface digitizing in forensic medicine: 3D documentation of skin and bone injuries. Forensic Sci Int 137(2-3):203-208.

https://doi.org/10.1016/j.forsciint.2003.07.009

12. Buck U (2005) 3D-Dokumentation und Visualisierung forensisch relevanter Befunde an Körperoberflächen und im Körperinneren. Der Vermessungsingenieur Heft 5:384-388

13. Campana L, Breitbeck R, Bauer-Kreuz R, Buck U (2016) 3D documentation and visualization of external injury findings by integration of simple photography in CT/MRI data sets (IprojeCT). Int J Legal Med 130(3):787-797. https://doi.org/10.1007/s00414-015-1274-3

14. Thali MJ, Dirnhofer R, Vock P (2009) The virtopsy approach. CRC Press USA, Boca Raton. https://doi.org/10.1201/9780849381898

15. Thali MJ, Braun M, Buck U, Aghayev E, Jackowski C, Vock P, Sonnenschein M, Dirnhofer R (2005) VIRTOPSY - scientific documentation, reconstruction and animation in forensic: individual and real 
3D data based geometric approach including optical body/object surface and radiological CT/MRI scanning. J Forensic Sci 50(2):428-442

16. Buck U, Naether S, Braun M, Bolliger S, Friederich H, Jackowski C, Aghayev E, Christe A, Vock P, Dirnhofer R, Thali MJ (2007) Application of 3D documentation and geometric reconstruction methods in traffic accident analysis: with high resolution surface scanning, radiological MSCT/MRI scanning and real data based animation. Forensic Sci Int 170(1):20-28.

https://doi.org/10.1016/j.forsciint.2006.08.024

17. Bolliger MJ, Buck U, Thali MJ, Bolliger SA (2012) Reconstruction and 3D visualisation based on objective real 3D based documentation. Forensic Sci Med Pathol 8(3):208-217.

https://doi.org/10.1007/s12024-011-9288-8

18. Buck U, Naether S, Rass B, Jackowski C, Thali MJ (2013) Accident or homicide-virtual crime scene reconstruction using 3D methods. Forensic Sci Int 225(1-3):75-84.

https://doi.org/10.1016/j.forsciint.2012.05.015

19. Subke J, Haase S, Wehner HD, Wehner F (2002) Computer aided shot reconstructions by means of individualized animated three-dimensional victim models. Forensic Sci Int 125(2-3):245-249.

https://doi.org/10.1016/S0379-0738(02)00007-5

20. Urschler M, Holler J, Bornik A et al (2014) Intuitive presentation of clinical forensic data using anonymous and person-specific 3D reference manikins. Forensic Sci Int 241:155-166.

https://doi.org/10.1016/j.forsciint.2014.05.017

21. Kersten TP, Przybilla HJ, Lindstaedt M, Tschirschwitz F, Misgaiski-Hass M (2016) Comparative geometrical investigations of hand-held scanning systems. Int Arch Photogramm Remote Sens Spat Inform Sci XLI-B5:507-514. https://doi.org/10.5194/isprsarchives-XLI-B5-507-2016

22. ATOS - Industrial 3D Scanning Technology. last accessed: 21-02-2017

http://www.gom.com/metrology-systems/atos.html

23. Portable 3D-Scanner: Go!SCAN 3D. last accessed: $21-02-2017$

https://www.creaform3d.com/en/metrology-solutions/handheldportable-3d-scanner-goscan-3d

24. Agisoft PhotoScan. last accessed: 21-02-2017 http://www.agisoft.com/

25. Artec Space Spider. last accessed: 21-02-2017 https://www.artec3d.com/3d-scanner/artec-spider

26. “Agisoft PhotoScan User Manual: Professional Edition, Version 1.2." (2016)

27. Lachat E, Macher H, Landes T, Grussenmeyer P (2015) Assessment and calibration of a RGB-D camera (Kinect v2 sensor) towards a potential use for close-range 3D modeling. Remote Sens 7(10):13070-13097. https://doi.org/10.3390/rs71013070

28. Buck U, Naether S, Kreutz K, Thali M (2011) Geometric facial comparisons in speed-check photographs. Int J Legal Med 125(6): 785-790. https://doi.org/10.1007/s00414-010-0518-5 


\section{Figures}
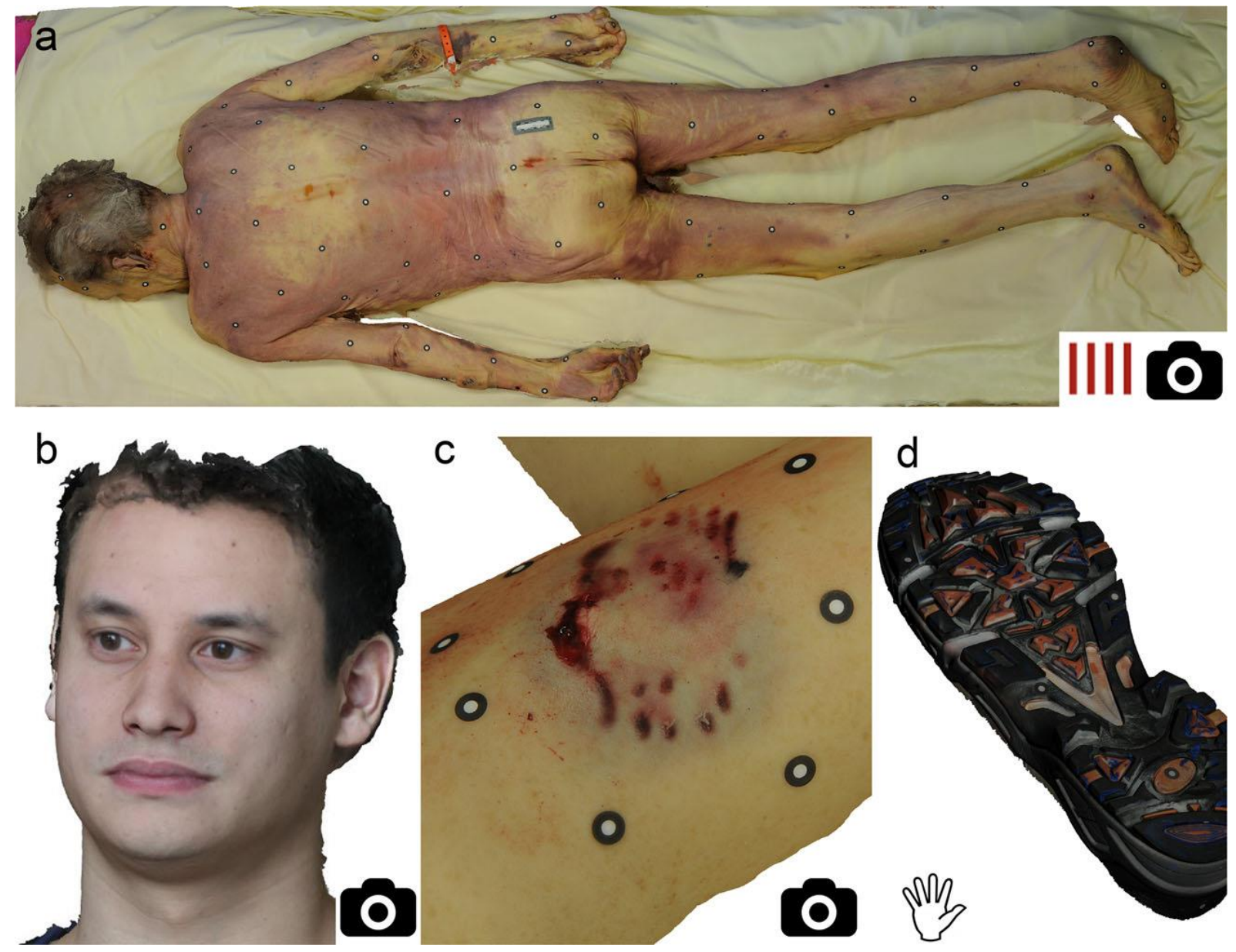

Fig. 1 3D models of the study objects (body of a deceased using ATOS III (icon: vertical red stripes) and PhotoScan (icon: camera) (a), face (b) and injury of living person using PhotoScan (c), and shoe using Space Spider (icon: hand) (d)) 

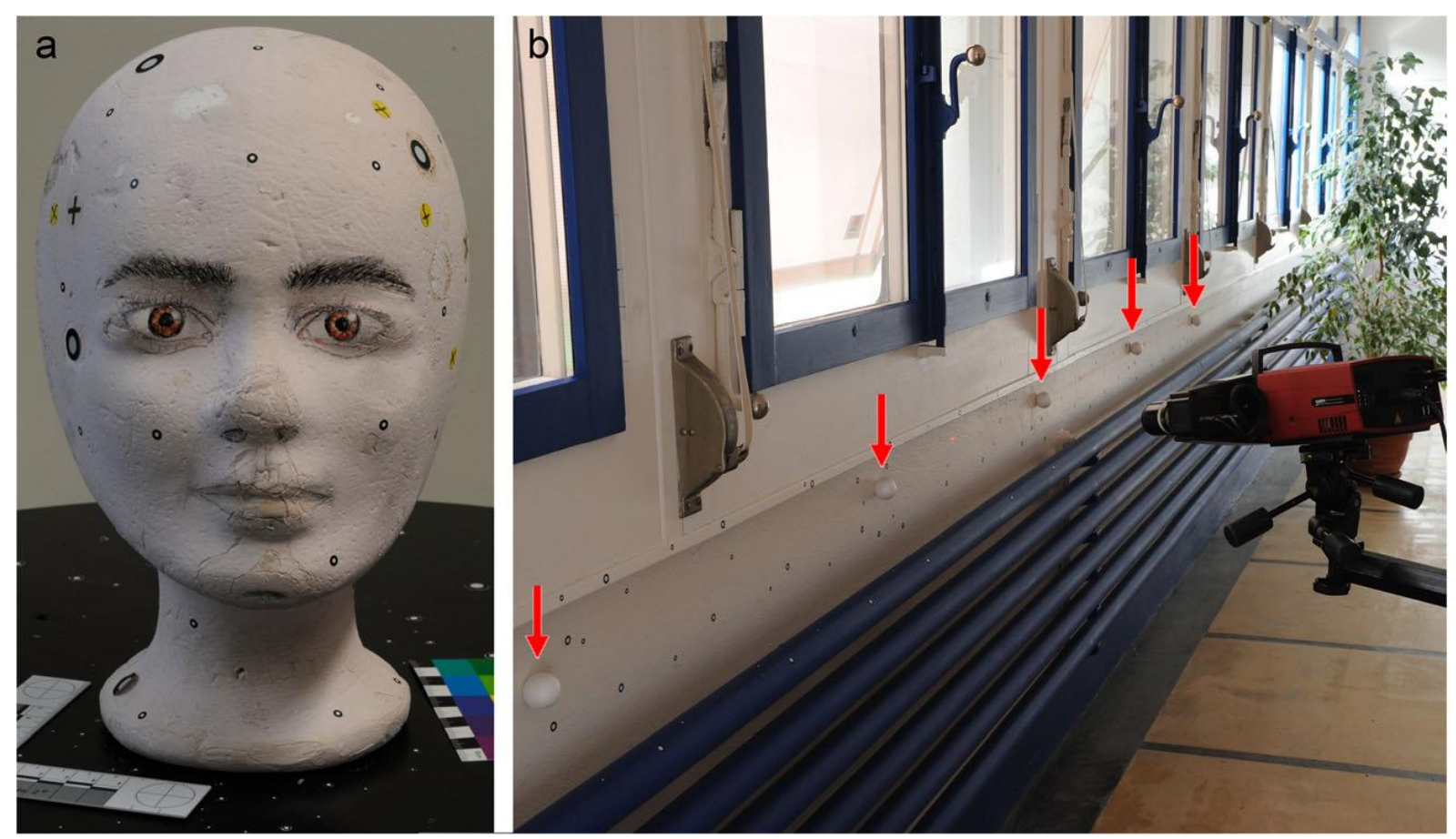

Fig. 2 Test objects created for checking the accuracy.

a Round test object: head of polystyrene.

b Long test object: $5 \mathrm{~m}$ wall with polystyrene spheres (red arrows) and ATOS III scanner
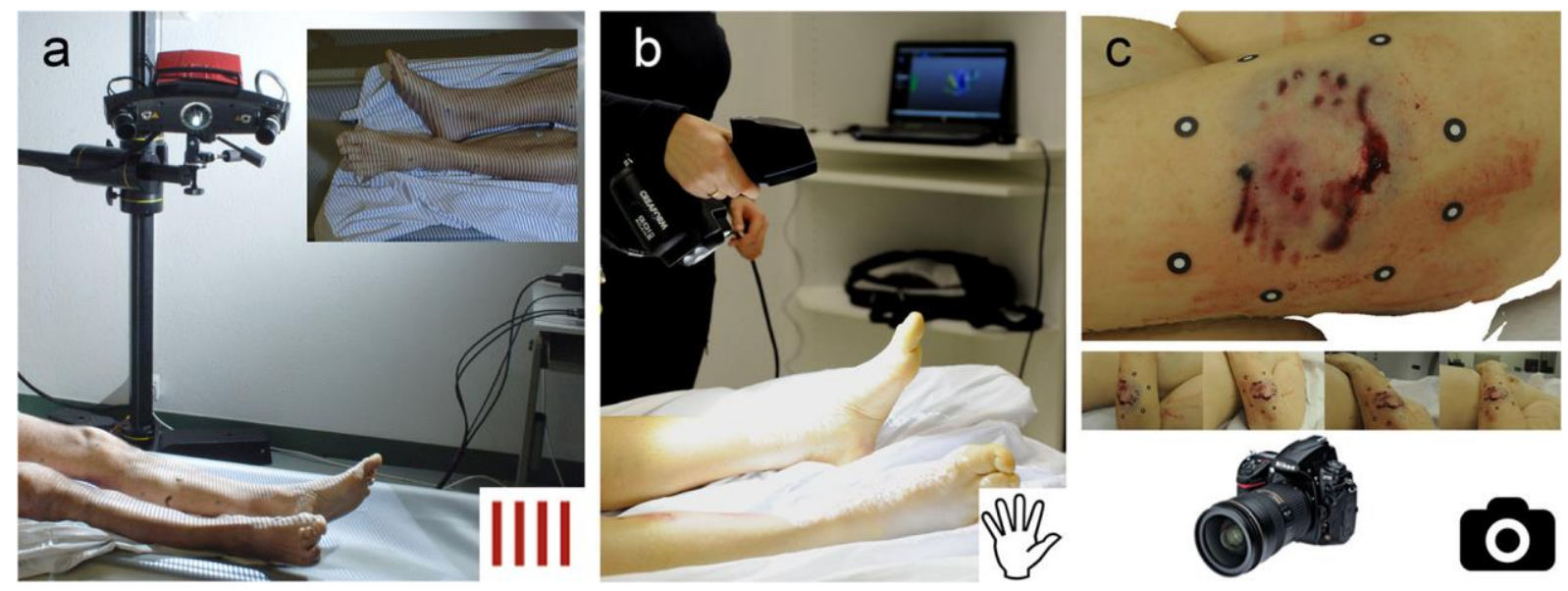

Fig. 3 Chosen 3D measuring systems within this study.

a Stripe-light scanner: GOM ATOS III.

b Hand-held scanner: Creaform Go!Scan 50 (icon: hand).

c Photogrammetry: PhotoScan 

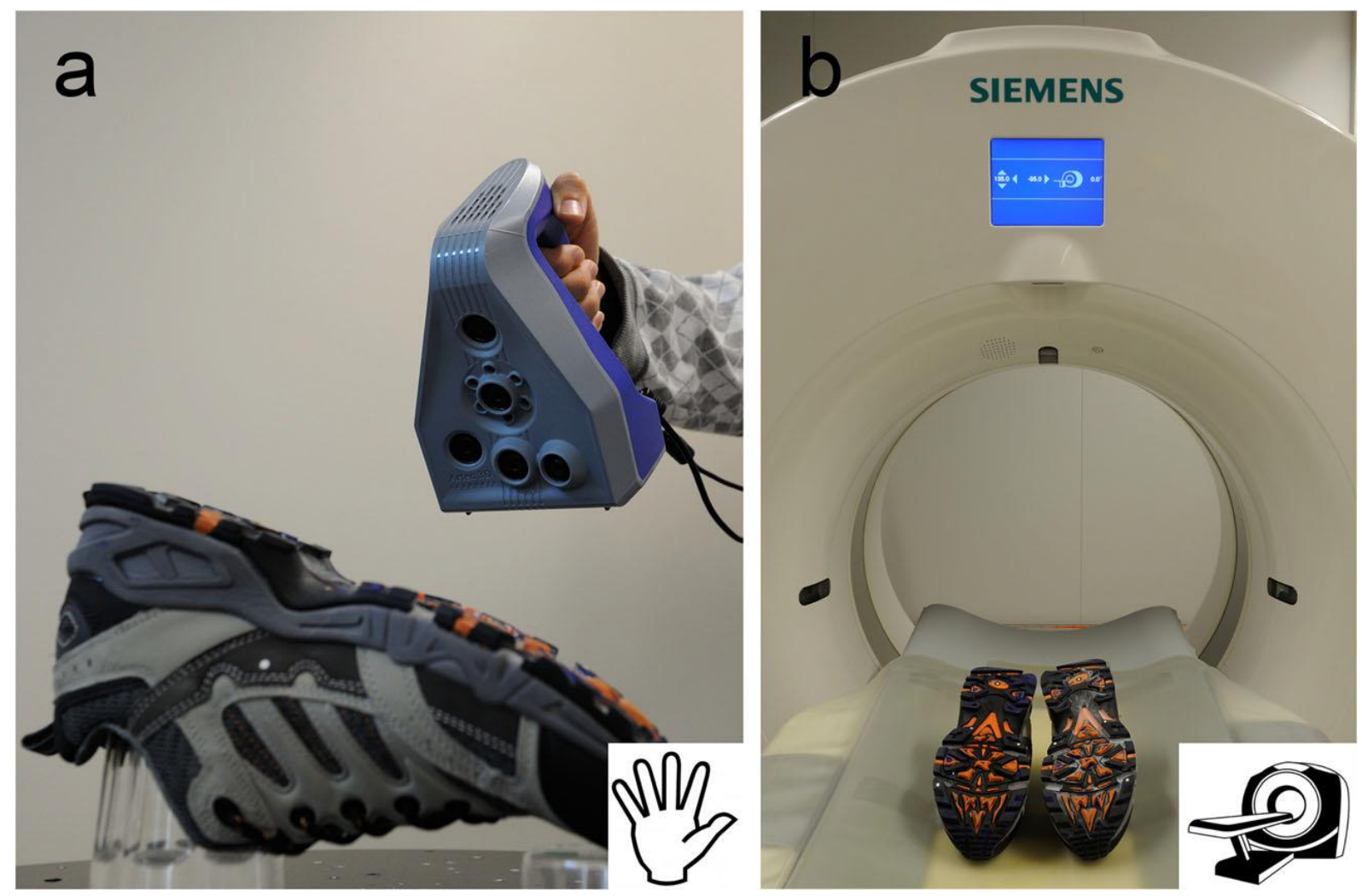

Fig. 4 Additionally chosen 3D measuring systems within this study just for the sole of the shoe.

a Hand-held scanner: Artec 3D Space Spider.

b Computed tomography (CT): Siemens Medical Somatom Definition AS (icon: CT) 

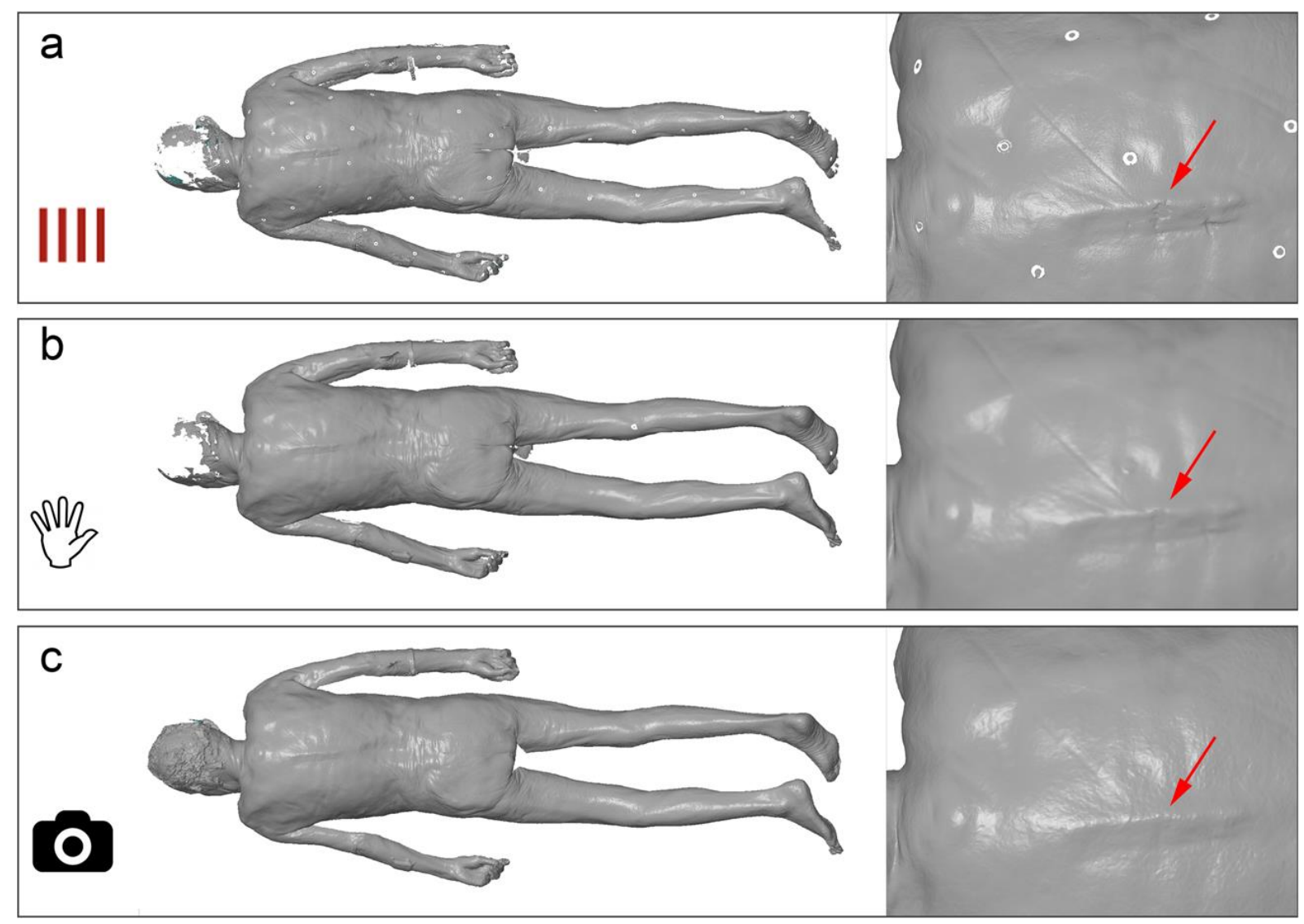

Fig. 5 Surface model of the body of a deceased and detail of his back generated with data from stripe-light scanner (a), hand-held scanner (b), and photogrammetry (c). Hairs are difficult to capture by scanners. The red arrows point at a skin lesion on the back. 

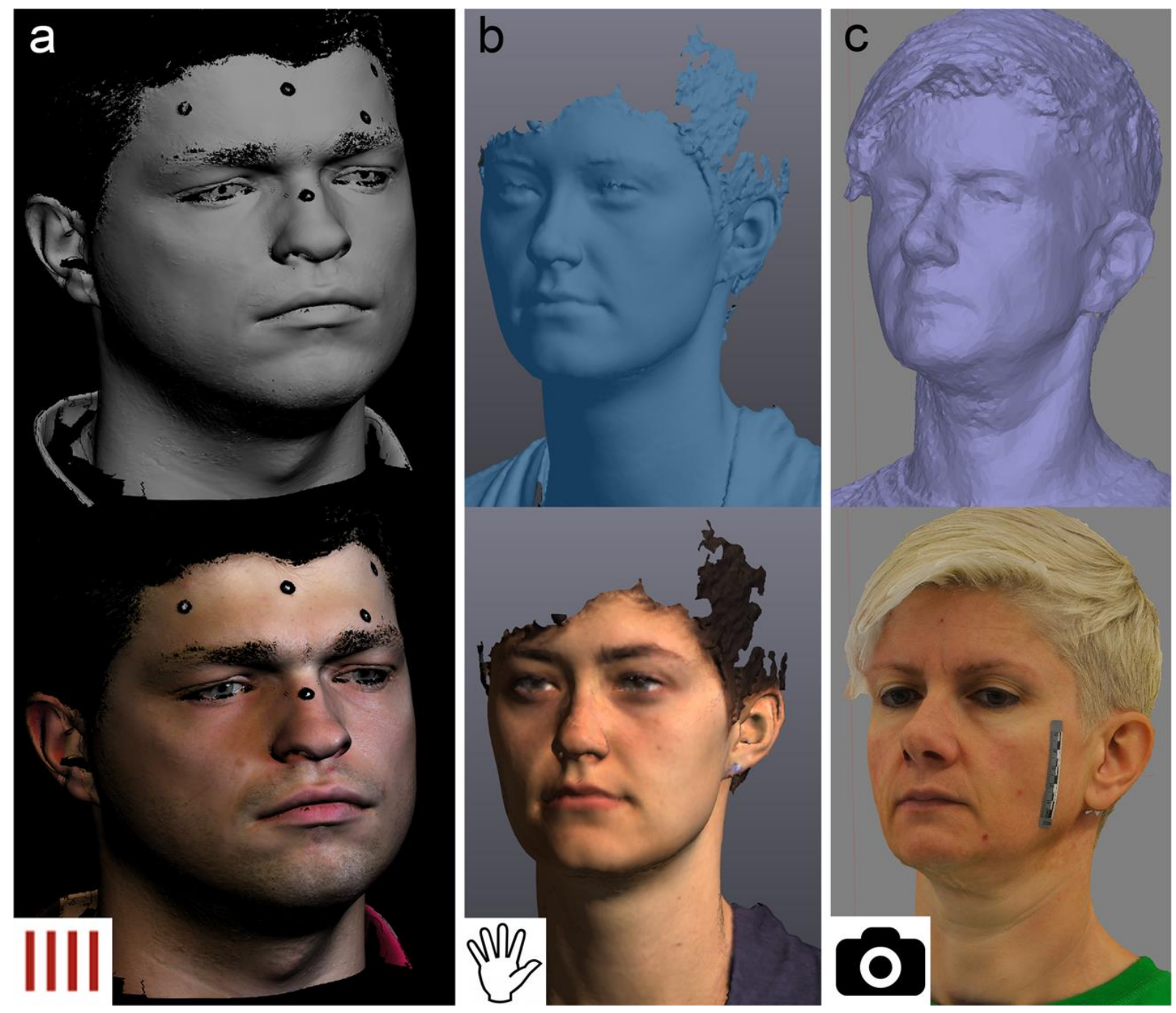

Fig. 6 3D model of a face generated with data from stripe-light scanner with projected photo (a), hand-held scanner (b), and photogrammetry (c). 

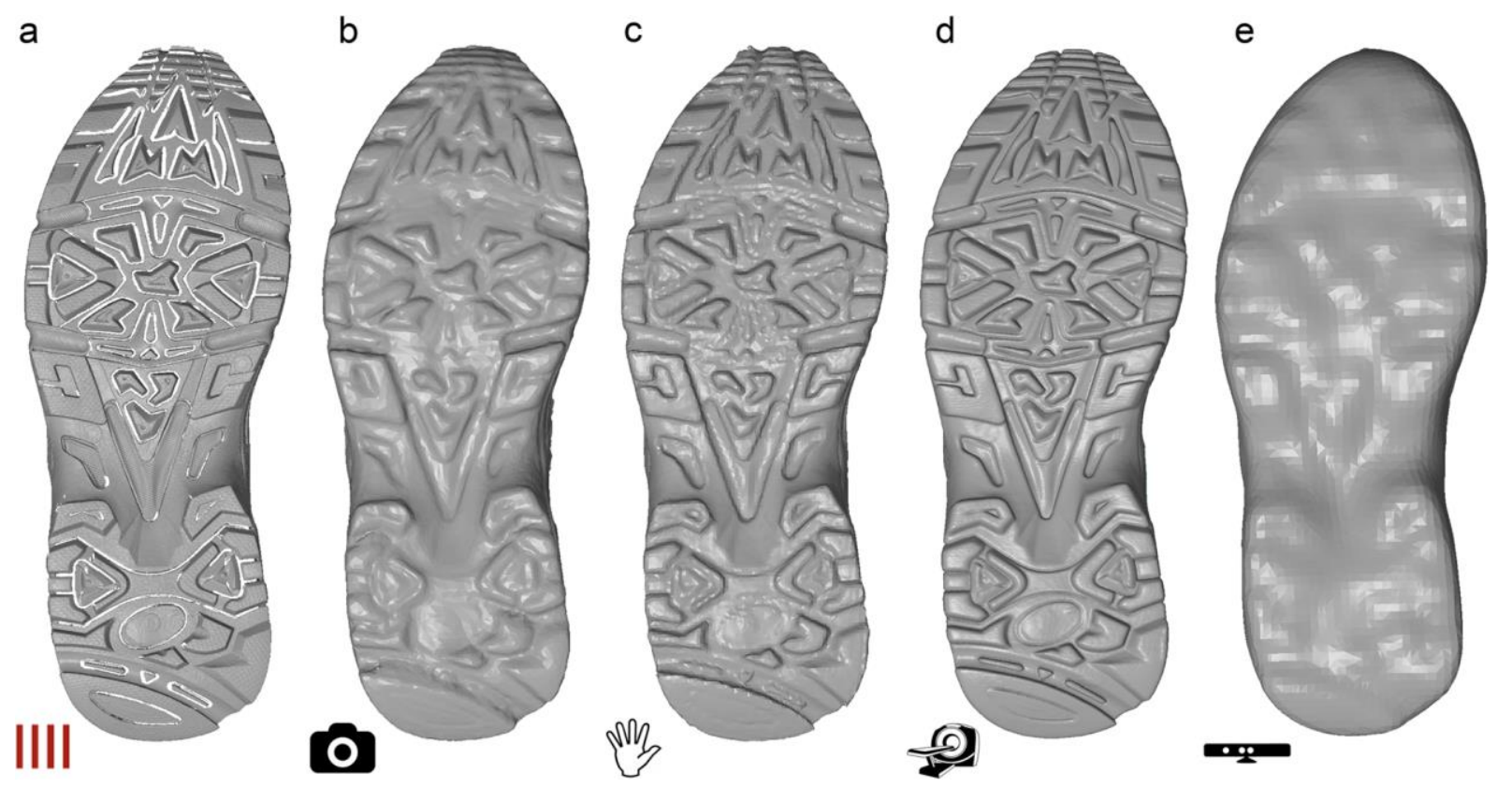

Fig. 7 3Dmodel of the shoe sole generated with data from stripe-light scanner (a), photogrammetry (b), hand-held scanner (c), CT (d), and Kinect sensor (icon: Kinect sensor) (e).
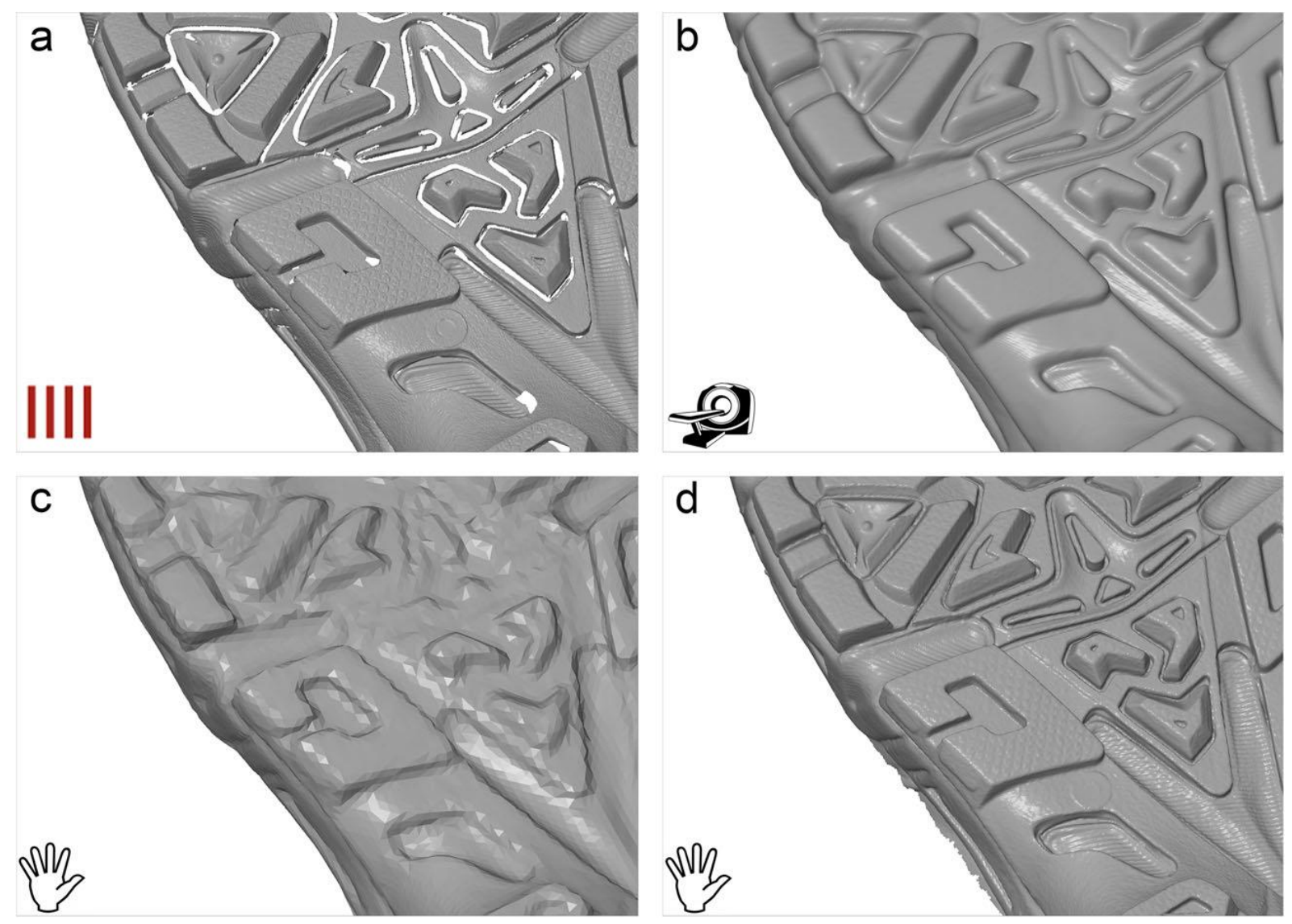

Fig. 8 Detail of the 3D model of the shoe sole generated with data from stripe-light scanner (a), CT (b), hand-held scanner (Creaform Go!Scan 50) (c), and high-resolution hand-held scanner (Artec 3D Space Spider) (d). 

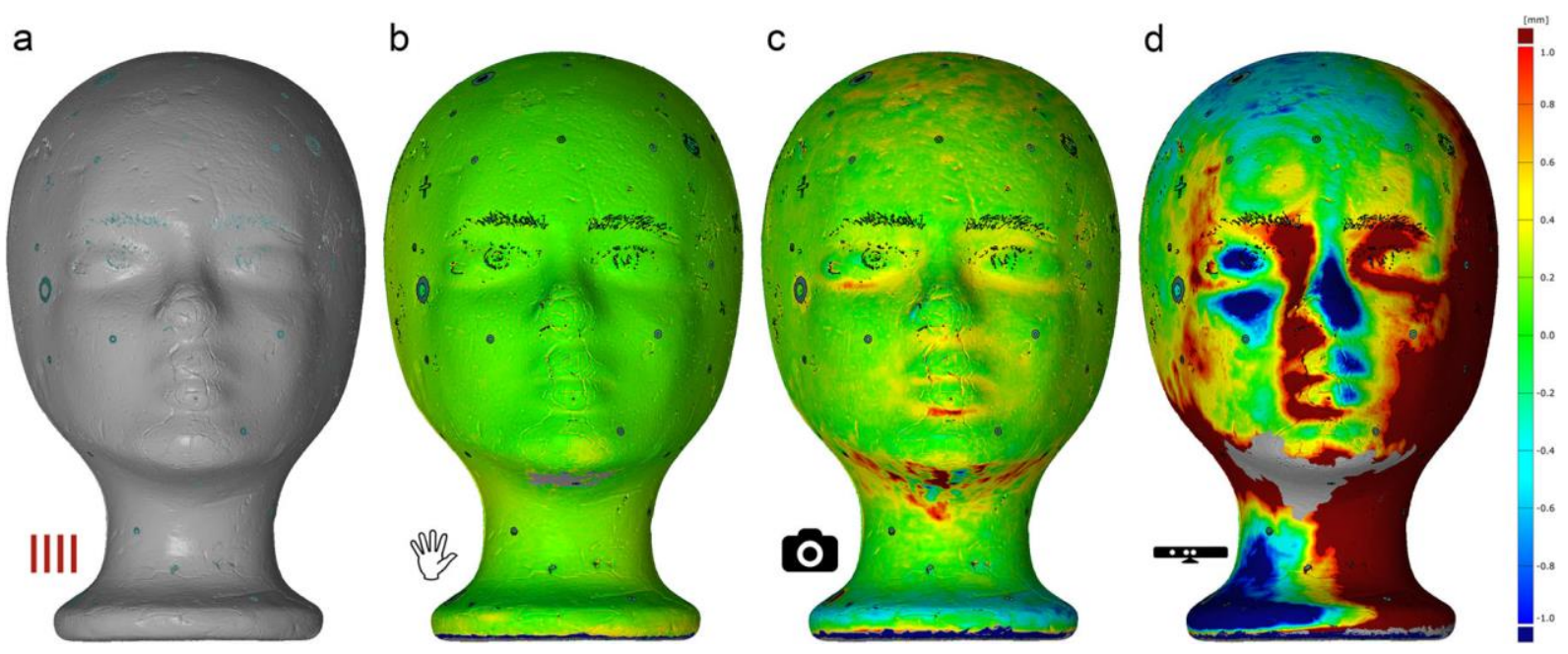

Fig. 9 Measurement deviation on round test object.

a Stripe-light scanner result as target.

b Target-actual-difference with hand-held scanner.

c Target-actual-difference with photogrammetry.

d Target-actual-difference with Kinect sensor 

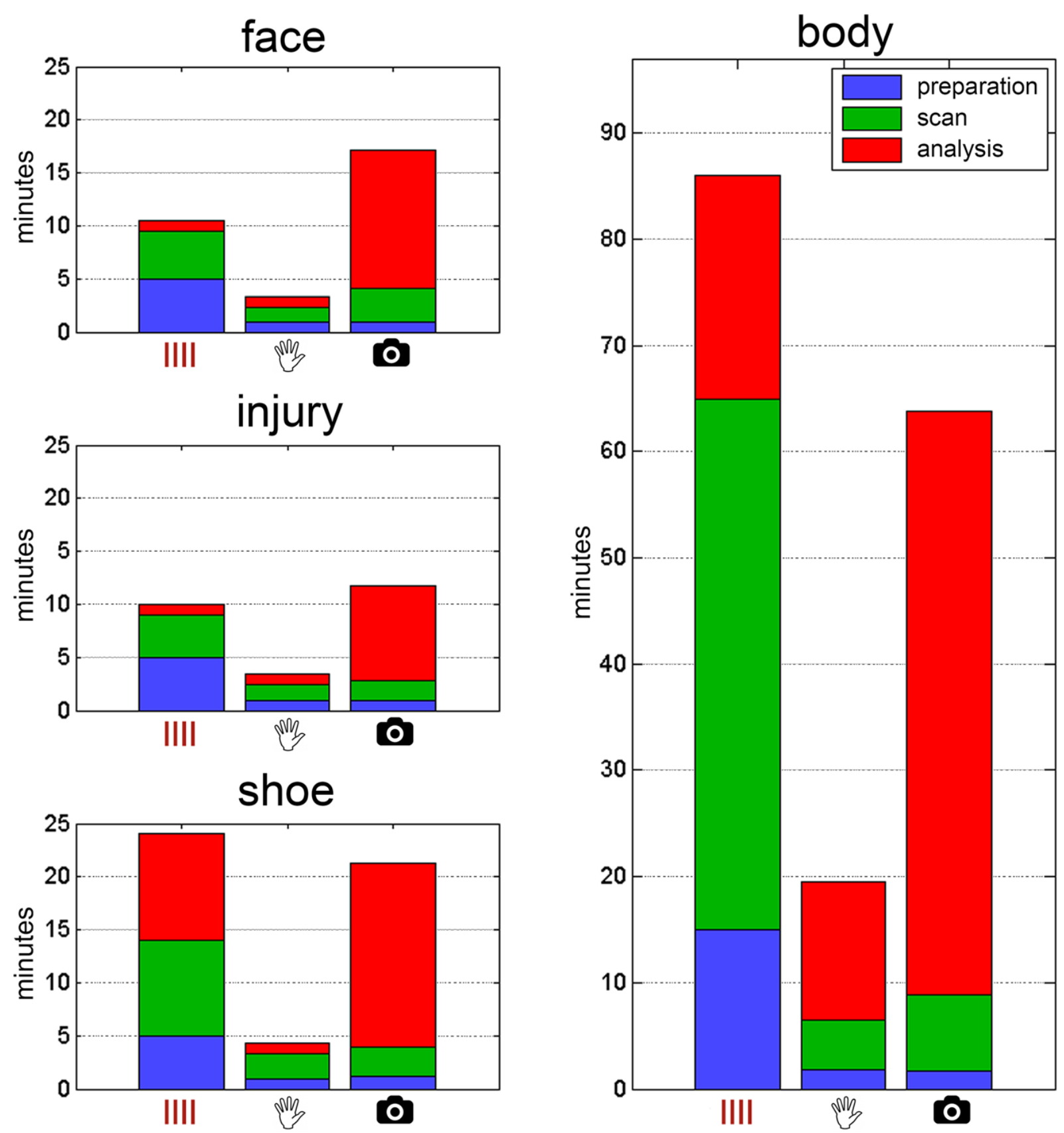

Fig. 10 Time-effort analysis 


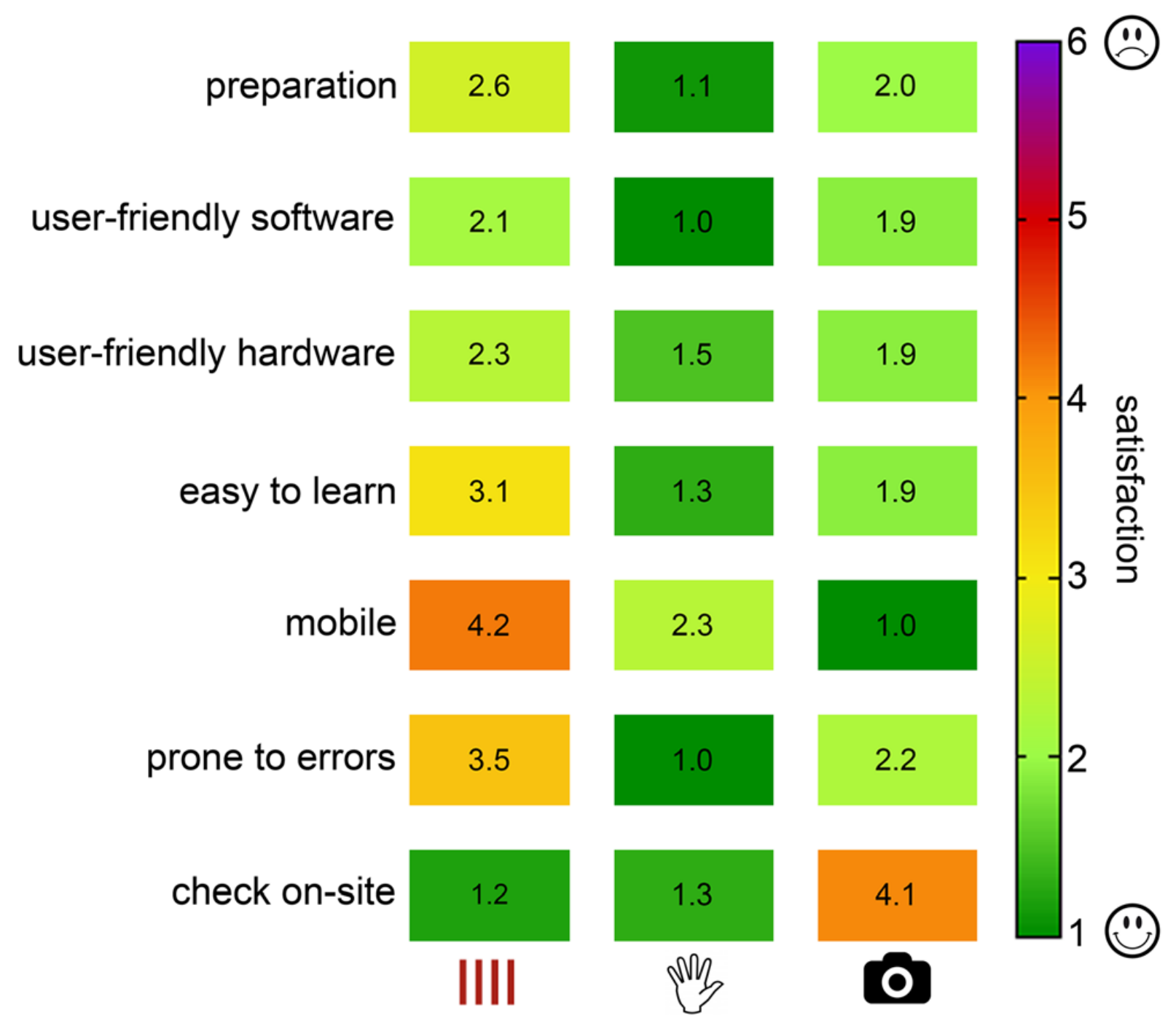

Fig. 11 Results of the questionnaire 